International Journal of Modern Physics A

(C) World Scientific Publishing Company

\title{
Lattice Chirality and its uses at large $N_{c}$
}

\author{
Rajamani Narayanan \\ Florida International University, Department of Physics, Miami, FL 33199. \\ email: rajamani.narayanan@fiu.edu \\ Herbert Neuberger* \\ Rutgers University, Department of Physics and Astronomy, Piscataway, NJ 08855. \\ email: neuberg@physics.rutgers.edu
}

\begin{abstract}
A brief overview of the authors' work on lattice chirality and its application to the numerical study of planar QCD is presented.

Keywords: Lattice Chirality; Large N
\end{abstract}

\section{Lattice Chirality}

Path integrals are formal objects. Sometimes one says that the integration measure is ill defined, to indicate that we do know the classical action, but, as we know quite well, classical Lagrangians simply are not guaranteed to produce unique quantum mechanical systems. Separating the problem into one of measure versus one of classical action is more a psychological step than a concrete mathematical statement; the fundamental construct is the quantum theory. The path integral needs to be defined as a whole, and the separation into a measure term and the rest is ambiguous.

In the mid 80's mathematical physicists provided a concrete view of the path integral over Weyl fermions in Euclidean space-times with non-abelian gauge backgrounds: The result of "performing" the fermion integral is not a function of the background, but, rather, a U(1) bundle defined over the space of all backgrounds. Anomalies indicate when the bundle can be trivialized. The bundle itself is defined over the space of gauge orbits and is therefore tautologically gauge invariant. Anomalies affect the choice of sections to the bundle: to do the remaining functional integrations one needs to pick a section.

Most lattice field theorists ignored these developments because anomalies were discovered and understood much earlier and without using anything close to the latter insight. Moreover, the new insight was specific to the Euclidean version of the theory. We know now that this was a mistake, but that was not obvious at the time. What one failed to appreciate was that the lattice made gauge invariance exact, and,

*Speaker at the International Conference on QCD and Hadronic Physics, June 16-20, 2005, Beijing. 
in the Euclidean action formalism, there was no escape from a more radical change of view about what a lattice fermion integral really meant: For example, an integral over an odd number of fermion fields had to be permitted to give a non zero answer!

The successful resolution of the problem of lattice chirality is rooted in a paper by Callan and Harvey who showed how anomalies on embedded defects are realized by current inflows. Later, David B. Kaplan showed that the Callan Harvey effect works on the lattice with Wilson fermions. Almost simultaneously with Kaplan, and completely independently, Frolov and Slavnov came up with the idea that in order to regulate a chiral gauge theory one needs a strictly infinite number of extra regulator fields. An old paper by Ginsparg and Wilson presciently observed that global chiral symmetries could be deformed on the lattice without losing any of their physical consequences and the modern development realized that observation in the case of QCD.

The objective is to define a chiral determinant line bundle on the lattice, over the space of all gauge orbits. The space of gauge orbits needs to have some subsets excised to permit the existence of nontrivial bundles. The infinite number of four dimensional fermion fields in the Callan and Harvey, David B. Kaplan and Frolov and Slavnov setups make the path integrals ambiguous to the extent that they do not always produce a gauge invariant function. However, they can always be used to gauge invariantly construct a line bundle.

In the vector like case the product of the bundle and its conjugate produces a function which turns out to be the determinant of a matrix that obeys the Ginsparg Wilson version of global lattice chirality. That matrix is called the "overlap" Dirac operator.

A U(1) bundle is a smooth collection of one dimensional complex vector spaces. A direct way to define one is via a set of linear equations, in which the parameters enter smoothly, and which have a one dimensional space of solutions, that is one less equation than unknowns. Such are the equations that define eigenvectors of a matrix. In our context the matrix is the Wilson Dirac operator with mass set to negative unity and r-parameter set to one, $H_{W}$. Close enough to continuum, $H_{W}^{2}$ is bounded away from zero and it is meaningful to define a projector on the subspace spanned by eigenvectors of $H_{W}, v_{i}$, with positive eigenvalues. For gauge fields that can be deformed to zero, the number of positive and negative eigenvalues of $H_{W}$ are equal. (If there is a difference, it gives the topological charge of the background.) Let us assume that the topological charge is zero. Let $w_{j}$ be the eigenvectors of $H_{W}$ with positive eigenvalues for a positive Wilson mass. The line bundle is given by:

$$
\operatorname{det}_{j, i}<w_{j} \mid v_{i}>
$$

The bundle is defined only in terms of projectors on the positive eigenspaces, $P_{+}$ and $P_{-}$, and therefore it is not surprising that the function giving the absolute value of the bundle can be expressed in terms of these projectors solely, or, equivalently, in terms of $\epsilon_{ \pm}=1-2 P_{ \pm}:\left|\operatorname{det}_{j, i}<w_{j}\right| v_{i}>\left.\right|^{2}=\operatorname{det} \frac{1+\epsilon_{+} \epsilon_{-}}{2}$.

The bundle can be nontrivial: One has to excise orbits for which $H_{W}$ has zero 
modes (the projectors are ill defined for those). One cannot deform smoothly $P_{-}$ by taking the Wilson mass to an extremal value to simplify $H_{W}$. However, $P_{+}$can be deformed and nothing is lost by replacing $\epsilon_{+}$by $\gamma_{5}$. $P_{-}$must remain nontrivial, with $\epsilon_{-}=\operatorname{sign}\left(H_{W}\right)$. The unitary matrix $V=\gamma_{5} \epsilon_{-}$becomes the central object.

The Nielsen Ninomiya theorem is avoided because one can use slightly different propagators for the sea and valence quarks. The sea quarks have a quadratic action with kernel usually denoted by $D_{o}$, the subscript standing for the letter $o$ from the word overlap $(\langle w \mid v\rangle)$. The valence quarks have a propagator that anticommutes with $\gamma_{5}$. It is highly nontrivial that one can use different propagators for the fermions in this manner. The proof rests on an argument in which one introduces an auxiliary field and declares that all Green's functions of interest are correlations of one particular linear combination of the fermion fields and the auxiliary field. The multiplicative contribution of the auxiliary field to the total fermion determinant is unity. In the continuum limit the auxiliary field is infinitely massive and there is no remnant of using the linear combination of fields as interpolating fields for the external quark lines.

The overlap Dirac operator $D_{0}=\frac{1+V}{2}$ is built out of the unitary matrix $V$, which is not sparse. The propagator on fermion lines connected to external quarks (valence quarks) is given by the gauge covariant matrix $A^{-1}$ which anticommutes with $\gamma_{5} . A^{-1}=\frac{1-V}{1+V}$.

The net practical result is that it becomes possible for the first time to establish $S \chi S B$ breaking at finite ultraviolet cutoff, independently of the approach to the continuum limit and any explicit chiral symmetry breaking.

\section{Uses at large $N_{c}$}

Eguchi Kawai reduction gave hope that one would be able to solve QCD at infinite $N_{c}$, still by numerical means, but faster than true QCD. The main stumbling block were lattice chirality and topology. These problems are now out of the way.

Consider YM at $N_{c}=\infty$ on a Euclidean torus of size $l^{4}$ (this space has a hypercubic symmetry group, preserved by typical lattice regularizations). There exists a physical length, $l_{c}$, such that traces of Wilson loops associated with loops $C, W(C)$, are exactly $l$ independent at $N_{c}=\infty$ for $l>l_{c}$. Hence, the finite $l$ looks infinite. For $l>l_{c}$, the entire extra symmetry group associated with an $S U\left(N_{c}\right)$ gauge theory on a four-torus, $Z^{4}\left(N_{c}\right)$, is unbroken and so is the hyper-cubic symmetry group.

On the continuum torus, momentum space is non-compact, but discrete, with gaps $2 \pi / l$. The eigenangles of Polyakov loops, $\theta_{\mu}^{i}$, contribute distinguishable momenta $\left|\Delta p_{\mu}^{i}\right|<2 \pi k_{\mu} /\left(N_{c} l\right)$, where $\left|k_{\mu}\right|<N_{c} / 2$. So long as the $\theta_{\mu}^{i}$ are uniformly distributed, the $\left|\Delta p_{\mu}^{i}\right|$ exactly fill the $2 \pi / l$ gaps. The $\theta_{\mu}^{i}$ will be uniformly distributed if the $Z^{4}\left(N_{c}\right)$ remains unbroken.

As $l$ is lowered to $l_{c}$, one of the four directions is picked randomly and the associated $Z\left(N_{c}\right)$ gets spontaneously broken. The transition is 1-st order. The eigenvalue spectrum of the Polyakov loop opens a gap at a random location. The infinite $N_{c}$ 


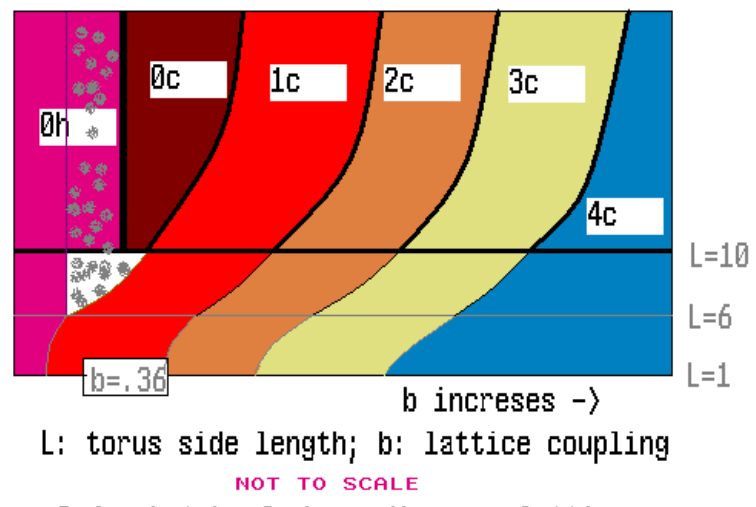

D=4, sketch of phase diagram; lattice

Fig. 1. A schematic description of the large $N$ phases of planar QCD on a four torus.

system suddenly "discovers" that one of the four directions is finite, but it still "believes" that the others are infinite. This has a precise meaning: the $N_{c}=\infty$ system represents finite temperature planar QCD, $T>l_{c}^{-1}$.

Figure 1 shows the various phases on the 4 dimensional lattice. 0h only exists on the lattice and disappears in the continuum. There are five different Xc phases, with $\mathrm{X}$ ranging from 0 to 4 . As $\mathrm{X}$ increases beyond 0 , sequentially, a Polyakov loop in one additional direction opens a gap, as sketched in Figure 2. The 0h to 0c transition is strongly first order and 0c extends into the 0h phase by metastability. The boundaries separating Xc phases satisfy asymptotic scaling and define separate critical sizes (exponential in the inverse gauge coupling). In all Xc phases, $\| W(1,1)-$ $1 \| \leq e$ where $W(1,1)$ is the smallest Wilson loop and $e<1$ approaches zero as $N_{c} \rightarrow \infty$; this produces an unambiguous definition of topology. There is no 0c phase for $L<5$ and in particular for $L=1$. That there is a 0 c phase for $L>4$ was missed in past work on reduction.

In Figure 3 the Monte Carlo data for the 0c to $1 \mathrm{c}$ transition is plotted and compared to tadpole improved asymptotic scaling, given by:

$$
b_{I}=b \frac{b^{2}-0.58960 b+0.08467}{b^{2}-0.50227 b+0.05479} ; \quad L_{c}(b)=(0.260 \pm 0.015)\left(\frac{11}{48 \pi^{2} b_{I}}\right)^{\frac{51}{121}} e^{\frac{24 \pi^{2} b_{I}}{11}}
$$

The massless Dirac operator takes the form $A=\left(\begin{array}{cc}0 & C \\ -C^{\dagger} & 0\end{array}\right)$ in the chiral basis with $C$ being a complex matrix. The size of $C$ is $n=2^{d / 2} L^{d} N_{c}$, where $d$ (even) is the dimension. Shuryak and Verbaarschot made $\mathrm{C}$ random, with enhanced symmetry: $p(C) d^{2 n^{2}} C \propto e^{-\kappa^{2} n \operatorname{Tr}\left(C^{\dagger} C\right)} d^{2 n^{2}} C$. The model correctly captures the distribution of the small eigenvalues of $C^{\dagger} C$, up to one scale determined by the chiral condensate.

It was determined by numerical simulation that the distribution of the two lowest eigenvalues of $-A^{2}$ indeed is given by RMT with a nonzero $\kappa$, at finite $L$ and infinite 


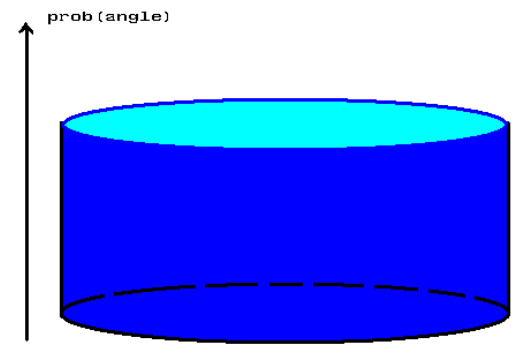

Spectrum of Polyakov Loop. Uniform.

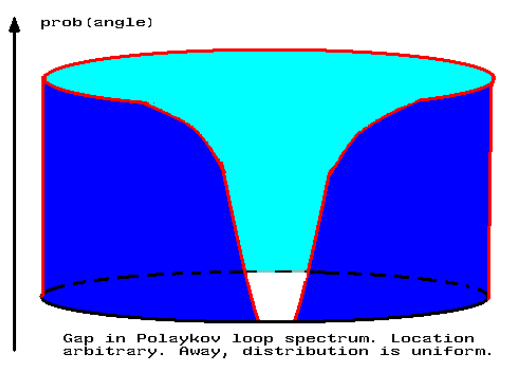

Fig. 2. A schematic illustration of the breaking of $Z\left(N_{c}\right)$ in some direction.

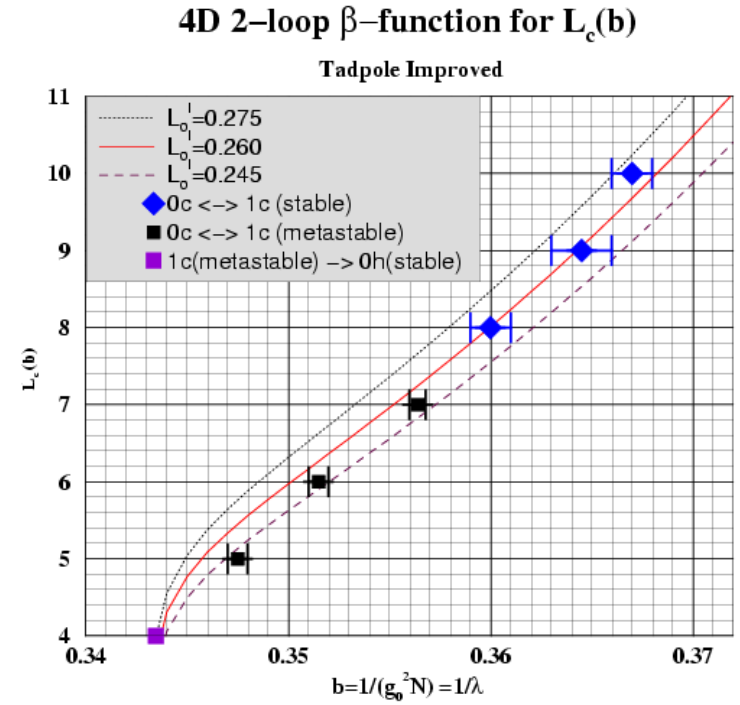

Fig. 3. A plot of the critical lattice size as a function of lattice coupling; obviously the size is an integer and the Monte Carlo simulation was used to find the corresponding gauge coupling $b$. 
$N_{c} . \kappa$ is independent of $L$. This establishes spontaneous chiral symmetry breaking and large $N_{c}$ reduction for the chiral condensate: $\frac{1}{N_{c}}\langle\bar{\psi} \psi\rangle=\kappa$.

In the two dimensional 't Hooft model (planar QCD) the meson mass, $m^{2}\left(m_{q}\right)$, contains enough information to almost reconstruct the full 't Hooft's meson equation. That equation can be "localized" by adding extra degrees of freedom. Broadly speaking, an extra dimension also localizes meson wave equations in AdS duals. Assume that four dimensional $N_{c}=\infty$ QCD is described by a dual with a warp factor, one scalar real function of one scalar real variable as conjectured by Polyakov. Could a meson probe determine the unknown warp function by something akin to inverse scattering, given $m^{2}\left(m_{q}\right)$ in the planar limit ? We analyzed the MC data using a dimensionless and renormalization group-invariant parameterization:

$$
\begin{gathered}
\Delta=\frac{1}{2}\left[\sqrt{m_{\pi}^{2} L_{c}^{2}(b)+\Lambda_{\pi}^{2}}-\Lambda_{\pi}\right] ; \quad \frac{1}{4} m_{\pi}^{2} L_{c}^{2}(b)=\Delta\left(\Delta+\Lambda_{\pi}\right) \\
\Delta=m_{o} \Sigma(b) L_{c}^{4}(b)+\frac{1}{\Lambda_{q}} m_{o}^{2} \Sigma^{2}(b) L_{c}^{8}(b)+\ldots
\end{gathered}
$$

Fits to the data produce: $\Lambda_{\pi}=6.91 \Lambda_{q}=1.03$. This also allows the extraction of the pion decay constant at infinite $N_{c}$ and we see that it has a large finite $\frac{1}{N_{c}}$ correction at $N_{c}=3: f_{\pi}(Q C D) \approx \sqrt{3} \frac{f_{\pi}\left(N_{c}=\infty\right)}{\sqrt{N_{c}}}=123 \mathrm{MeV} \neq 86 \mathrm{MeV}$.

At $N=\infty m^{2}\left(m_{q}\right)$ is well described by a parabola for all $m_{q}$, well beyond the range of chiral perturbation theory. The parameter $\Delta$ enters in a way familiar from mass formulae obtained using the supergravity dual to the planar limit of certain field theories, but no longer is defined in terms of a dimension. This and the observations about the 't Hooft model lead to the question: Does the parameterization

of $m^{2}$ in terms of a $\Delta$ indicate that the meson wave equation can be approximately localized via the introduction of extra degrees of freedom?

\section{Acknowledgments}

This research was partially supported by the DOE under grant number DE-FG0201ER41165 at Rutgers University and by the NSF under grant number PHY-030065 at FIU. Herbert Neuberger is grateful to the conference organizers for the invitation.

\section{References}

This list consists of the authors' papers hereby reviewed and omits many other important contributions because of space limitations.

1. R. Narayanan, H. Neuberger, Phys. Lett. B302 (1993) 62.

2. R. Narayanan, H. Neuberger, Nucl. Phys. B412 (1994) 574.

3. R. Narayanan, H. Neuberger, Nucl. Phys. B443 (1995) 305.

4. H. Neuberger, Phys. Lett. B427 (1998) 353.

5. H. Neuberger, Phys. Rev. Lett. 81 (1998) 4060.

6. H. Neuberger, Phys. Rev. D59 (1999) 085006.

7. J. Kiskis, R. Narayanan, H. Neuberger, Phys. Lett. B574 (2003) 65.

8. R. Narayanan, H. Neuberger, Nucl. Phys. B696 (2004) 107.

9. R. Narayanan, H. Neuberger, Phys. Lett. B616 (2005) 76. 